Check for updates

Cite this: RSC Adv., 2017, 7, 35490

Received 27th April 2017

Accepted 1st July 2017

DOI: $10.1039 / c 7 r a 04752 e$

rsc.li/rsc-advances

\title{
Investigations on the existence of piezoelectric property of a bio-polymer - chitosan and its application in vibration sensors
}

\begin{abstract}
E. Praveen, ${ }^{a}$ S. Murugan ${ }^{b}$ and K. Jayakumar (D) *a
A variety of physical and chemical properties of bio-polymers like chitosan have been investigated although their inherent behavior, such as piezoelectricity, has not been investigated to date. In this study, an attempt has been made to investigate the presence of non-centrosymmetry in chitosan through X-ray diffraction (XRD) analysis and second harmonic generation ( $\mathrm{SHG}$ ) experiment. Existence of piezoelectricity is established by determining $d_{33}$ coefficient for various pressures. With the help of the above mentioned results, a new application of the bio-polymer chitosan as a piezoelectric element in vibration sensors has been analyzed. To fabricate vibration sensor elements, chitosan was dissolved in various organic acids and its suitability for these applications was studied. Through isolation resistance measurement, the fabricated sensor is found to exhibit very high isolation, which is suitable even in hazardous environments. All the abovementioned investigations reveal the suitability of using chitosan in vibration sensors.
\end{abstract}

\section{Introduction}

The family of polymers, such as chitin and chitosan, possesses a variety of physical and chemical properties. In nature, chitin is found in the exoskeletons of arthropods and insects, shells of molluscs, pens and beaks of cephalopods, and cell-wall fungi such as Mucoraceae. Commercially, chitin and its derivatives, such as chitosan, are isolated from shells of crab, shrimp, prawn etc. via conventional steps such as demineralization, deproteinization, decolouration and deacetylation. ${ }^{1}$ Chitosan is the second most generous natural polysaccharide after cellulose on earth. These kind of resources are estimated to be available in $\sim 100$ billion tons in nature. ${ }^{2}$ Contrary to chitin, both amino and hydroxyl groups of chitosan can be selectively modified for versatile applications. Chitosan exhibits several properties such as biocompatibility, biodegradability, non-toxicity, and easily film formation ability that are advantageous for industrial and biomedical applications such as water treatment, ${ }^{3}$ dye removal, ${ }^{4}$ tissue engineering, ${ }^{5}$ wound healing and dressing, ${ }^{6,7}$ and drug delivery. ${ }^{8}$ Silva $e t$ al. have reported that the addition of chitosan to collagen polymer ${ }^{9}$ exhibits a considerable increment in the piezoelectric strain constant $\left(d_{14}\right)$. Recently, ferroelectric materials such as $\mathrm{ZnO}$ and $\mathrm{BaTiO}_{3}$ exhibiting piezoelectric property were embedded in chitosan polymer and used for the detection of mechanical excitation, where the piezoelectric nature of $\mathrm{ZnO}$

${ }^{a}$ Nanostructure Lab, Department of Physics, The Gandhigram Rural Institute-Deemed University, Gandhigram-624 302, Tamilnadu, India.E-mail: kjkumar_gri@rediffmail. com

${ }^{b} I P R C-I S R O$, Mahendragiri - 627 754, Tamilnadu, India and $\mathrm{BaTiO}_{3}$ was focused on and the electret was tested for mechanical sensing applications. ${ }^{10,11}$ In the present study, an attempt has been made to investigate the existence of noncentrosymmetry in chitosan through second harmonic generation (SHG) and that of piezoelectric property by measuring the piezoelectric strain coefficient $\left(d_{33}\right)$. After the analysis of SHG and $d_{33}$ data, isolation measurements of the chitosan samples are conducted by $80 \mathrm{~Hz}$ mechanical excitation. Sensitivity and selectivity of chitosan dissolved in various organic acids were studied and have been reported.

\section{Experimental techniques}

Chitosan used in the preparation of sensor elements was of analytical grade and procured from Pelican Biotech and Chemical Labs, India, and organic acids (such as formic, acetic, adipic, and succinic acids) were procured from $\mathrm{CDH}$, India, without further purification for the investigations. The degree of deacetylation (DDA) of chitosan was $91.2 \%$. The flakes of chitosan were fine grained with formic acid in an agate mortar that was air dried for few hours, and pellets of thickness $1.3 \mathrm{~mm}$ were prepared using the manual hydraulic pressure unit for the piezoelectric strain coefficient $\left(d_{33}\right)$ measurement. Since filmcasting method is a very simple and cost-effective technique for making freestanding films of chitosan, casting technique has been adopted for the preparation of samples. Thin films of chitosan were prepared by dissolving chitosan in formic, acetic, adipic, and succinic acids in separate flasks with an optimum concentration of $1.5 \%$ and stirred using a mechanical stirrer. The percentage of acid used for dissolving chitosan was 
optimized at $0.1,0.25,0.5,0.75,1.0,1.25$, and $1.5 \%$. As freestanding films of chitosan are necessary for vibration sensing measurement, the transparent solution was accurately weighed and cast on glass Petri dishes of equal area. The samples were air dried and undisturbed for 72 hours. Samples that were cast below the concentration of $1.5 \%$ were highly reedy, sticking to the substrate, and unable to peel off. Hence, finally, thin films of $30 \mu \mathrm{m}$ were peeled off from the substrate, and the solvent concentration $1.5 \%$ was chosen for vibration sensing analysis. However, thin films prepared by dissolving chitosan in formic acid are flexible and user friendly, whereas those prepared in acetic, adipic, and succinic acids are brittle and less stable and hence cannot be utilized as an element for the vibration sensing. Thus, chitosan dissolved in formic acid is highly focused in this study. Further, vibration sensing nature of these films is compared with that of the films obtained by dissolving chitosan in other organic acids such as acetic, adipic, and succinic acid.

\section{Results and discussion}

\section{Structural analysis of chitosan}

X-ray diffraction analysis. XRD profiles of chitosan and formate of chitosan (FC) are shown in Fig. 1a. The XRD spectrum of FC exhibits five major peaks at $11.9^{\circ}, 19^{\circ}, 23.4^{\circ}, 31.2^{\circ}$, and $38^{\circ}$ in the amorphous background of chitosan, which agrees with the study conducted by Ogawa et al. ${ }^{12}$ and reveals the hydrated nature and eight-fold helical conformation of chitosan, whereas the unreacted chitosan fragment has an extended two-fold helix. The reflection peaks are due to ionic

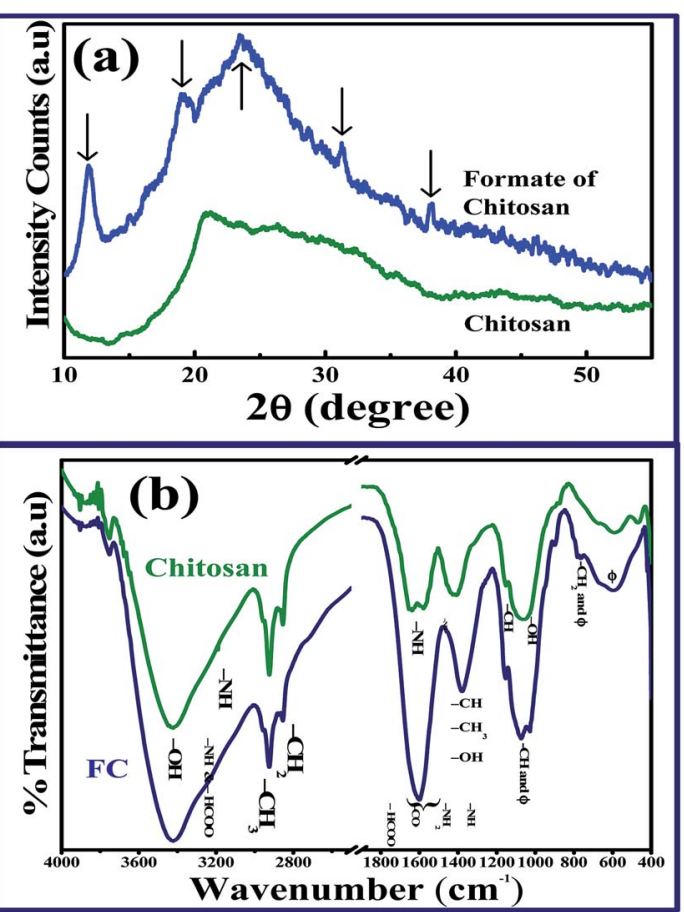

Fig. 1 (a) XRD profile and (b) FTIR spectra of chitosan and formate of chitosan. crosslinking of chitosan, confirming the increase in the crystallinity of the sample. The crystallinity of chitosan decreases with the increase in DDA, ${ }^{13}$ which exhibits a broad hump around $23.4^{\circ}$, confirming the semicrystalline nature of the sample. The sharp peak for $\mathrm{FC}$ at $11.9^{\circ}$ and the weak peak at $19^{\circ}$ confirm the hydrated nature of the sample. The broad absorption is elevated to $20.8^{\circ}$ for the pure chitosan sample, which endorses the amorphous nature of the chitosan matrix. ${ }^{\mathbf{1 4}}$ Hence, on comparing the XRD profile of chitosan and FC, the influence of the formic acid anion on the chitosan matrix was established. Considering the structural properties of chitosan, there are only two possible space groups ${ }^{15}$ available: $P 2_{1} 2_{1} 2_{1}$ and $P 2{ }_{1} 2_{1} 2$. Biological macromolecules, such as chitosan, consist of building blocks such as glucosamine, and in general, these building block structures are asymmetrical and their mirror reflections do not exist in nature. Hence, space groups with mirror reflection or inversion centres are not allowed for crystals of these molecules. From the data of occurrence of the space group for the protein crystal, probability of occurrence of $P 2_{1} 2_{1} 2_{1}$ (ref. 16) is larger than that of $P 2_{1} 2_{1} 2$. Therefore, the most favourable space group of chitosan is chosen as $P 2{ }_{1} 2_{1} 2_{1}$. Among the orthorhombic subgroups, cubic space groups of classes 23 and 432, which contain $P 22_{1} 2_{1} 2_{1}$, are non-centrosymmetric, ${ }^{17}$ where the origin is chosen in a way that it is surrounded by three pairs of $2_{1}$ axes. This principle maintains the aforementioned orthorhombic subgroups. In this study, for the first time, an attempt has been made to investigate the piezoelectric property of chitosan by considering its orthorhombic structure with $P 2{ }_{1} 2_{1} 2_{1}$ space group, thus confirming the existence of non-centrosymmetry, and chitosan is tested for its application in vibration sensors.

Infrared active modes of chitosan. Room-temperature Fourier transform infrared spectra (FTIR) were obtained in the range of $4000-400 \mathrm{~cm}^{-1}$ using Perkin Elmer LS 55 spectrometer with $2 \mathrm{~cm}^{-1}$ resolution. To avoid the presence of residual water content in the sample, all the samples were dried and used for the measurements. The spectra were obtained using $\mathrm{KBr}$ pellets prepared at a pressure of $740 \mathrm{MPa}$. In the IR spectra of chitosan, the broad absorption band at $3422 \mathrm{~cm}^{-1}$ corresponds to the stretching mode of the free hydroxyl group $(-\mathrm{OH})$ since the structure of pure chitosan is stabilized by interand intramolecular hydrogen bonds. When glucosamine units in chitosan are protonated by formic acid, the hydrogen bond involving the amide group $\left(-\mathrm{NH}_{2}\right)$ is shifted by $14 \mathrm{~cm}^{-1}$, as shown in Fig. 1b. Hence, the physical attachment of formate to the amide group of chitosan is confirmed. The IR active modes of chitosan are provided in Table 1.

Scanning electron microscopy analysis. On dissolving chitosan in formic, acetic, adipic, and succinic acids, different morphological changes were observed via scanning electron microscopy (SEM). Chitosan dissolved in formic acid exhibits a smooth surface unlike that dissolved in acetic, adipic, and succinic acids. Further, thickness of the film is measured and depicted in Fig. 2.

Second harmonic generation (SHG) of chitosan. Typical confirmation of the existence of non-centrosymmetry of the system was further tested via generation of second harmonic 
Table 1 IR active modes of chitosan and formate of chitosan and their respective vibrations in $\mathrm{cm}^{-1 a}$

\begin{tabular}{|c|c|c|c|c|c|}
\hline S. No. & Wavenumber ${ }^{13,18-20}(\bar{\lambda})$ & \multicolumn{2}{|l|}{ Chitosan } & \multicolumn{2}{|c|}{ Formate of chitosan } \\
\hline 2 & 3196 & 3340 & $\nu(\mathrm{NH})$ & 3354 & $\nu(\mathrm{NH})+\nu(\mathrm{HCOO})$ \\
\hline 3 & 2919 & 2924 & $\nu\left(\mathrm{CH}_{3}\right)$ & 2926 & $\nu\left(\mathrm{CH}_{3}\right)$ \\
\hline 4 & 2875 & 2872 & $\nu\left(\mathrm{CH}_{2}\right)$ & 2874 & $\nu\left(\mathrm{CH}_{2}\right)$ \\
\hline 7 & 1627 & 1638 & $\delta(\mathrm{NH})$ chitin & & \\
\hline 8 & 1565 & 1578 & $\delta\left(\mathrm{NH}_{2}\right)$ & & \\
\hline 9 & 1422 & 1424 & $\delta(\mathrm{CH})+\delta(\mathrm{OH})$ & 1380 & $\delta(\mathrm{CH})+\delta(\mathrm{OH})+\delta\left(\mathrm{CH}_{3}\right)$ \\
\hline 10 & 1417 & 1410 & $\delta\left(\mathrm{CH}_{3}\right)+\delta(\mathrm{CH})$ chitin & & \\
\hline 11 & 1156 & 1150 & $\delta(\mathrm{CH})+\delta(\mathrm{OH})$ & 1154 & $\delta(\mathrm{CH})+\delta(\mathrm{OH})$ \\
\hline 12 & 1075 & 1070 & & 1076 & \\
\hline 17 & 465 & 474 & $\delta(\mathrm{COC})$ chitin & & \\
\hline
\end{tabular}

signals. Hence, chitosan flakes were verified for SHG with excitation of $1064 \mathrm{~nm}$ using a Nd:YAG laser. The laser power used was typically less than $100 \mathrm{~mW}$ to avoid local heating of the sample. The generated second harmonic signals were compared with those of potassium diphosphate (KDP) crystal as a reference. Intensity of SHG signals generated for chitosan and formate of chitosan were $90 \%$ and $86 \%$ with respect to the KDP crystal, respectively. Although there is a small reduction in the SHG signal of chitosan dissolved in formic acid, the noncentrosymmetry is not altered. Hence, the existence of noncentrosymmetry in chitosan is well established, which will provide a new way for the fabrication of devices such as piezoelectric pressure sensors. SHG signals of chitosan and formate of chitosan are depicted in Fig. 3a with the comparison of the Nd:YAG source and KDP reference. Normalized SHG intensities and SHG intensity at $532 \mathrm{~nm}$ for chitosan dissolved in formic, acetic, succinic, and adipic acids are shown in Fig. $3 \mathrm{~b}$ and $\mathrm{c}$, respectively. Fig. $3 \mathrm{~b}$ and $\mathrm{c}$ show that the intensity of the SHG signal is more pronounced $532 \mathrm{~nm}$ for chitosan dissolved in formic acid than that for chitosan dissolved in other organic acids, indicating the manifestation of the non-centrosymmetric nature.

Piezoelectric coefficient $\left(\boldsymbol{d}_{33}\right)$ of chitosan. Piezoelectric strain coefficient of formate of chitosan is measured for various

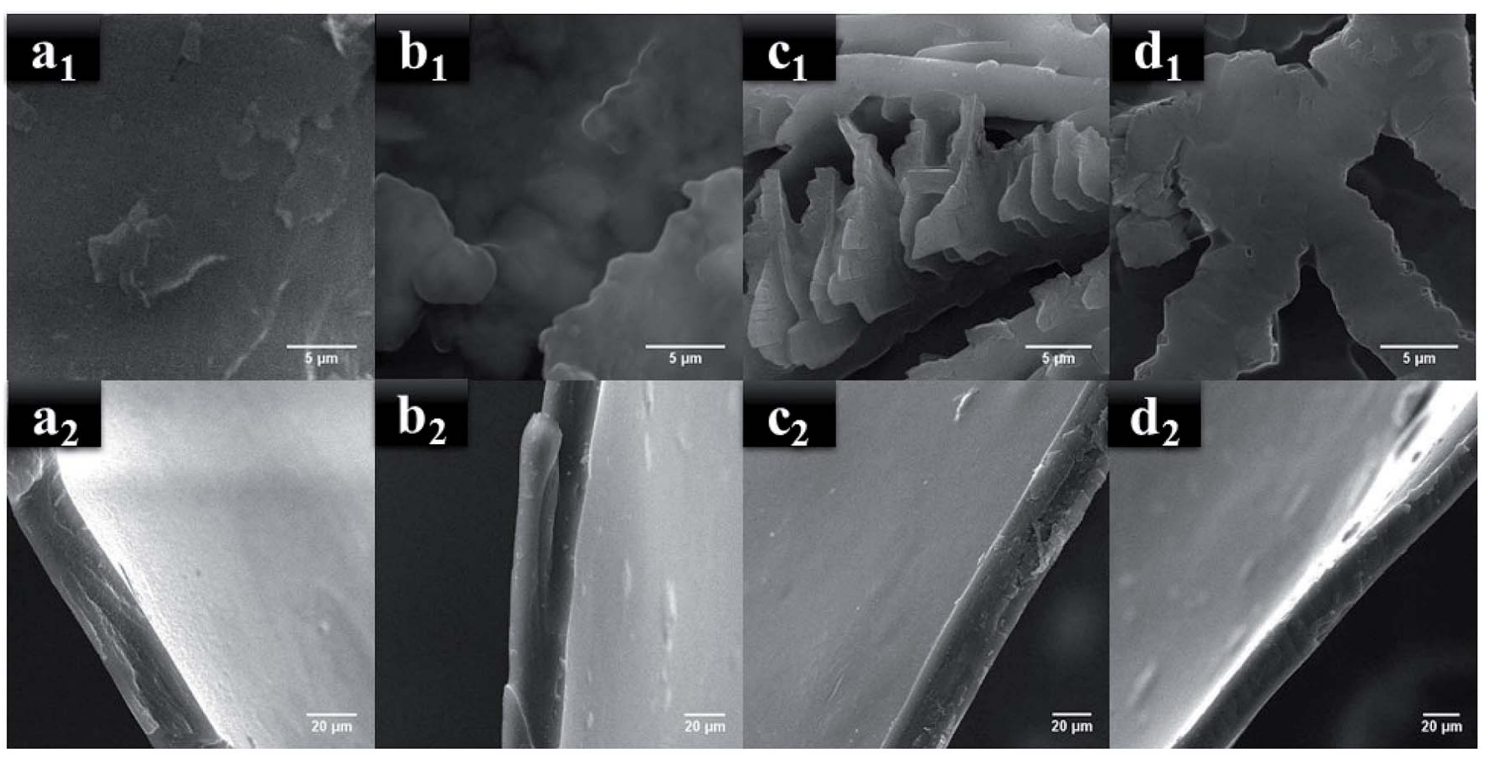

Fig. 2 Scanning electron images with top and cross sectional views of chitosan dissolved in (a) formic (b) acetic (c) succinic and (d) adipic acids. 

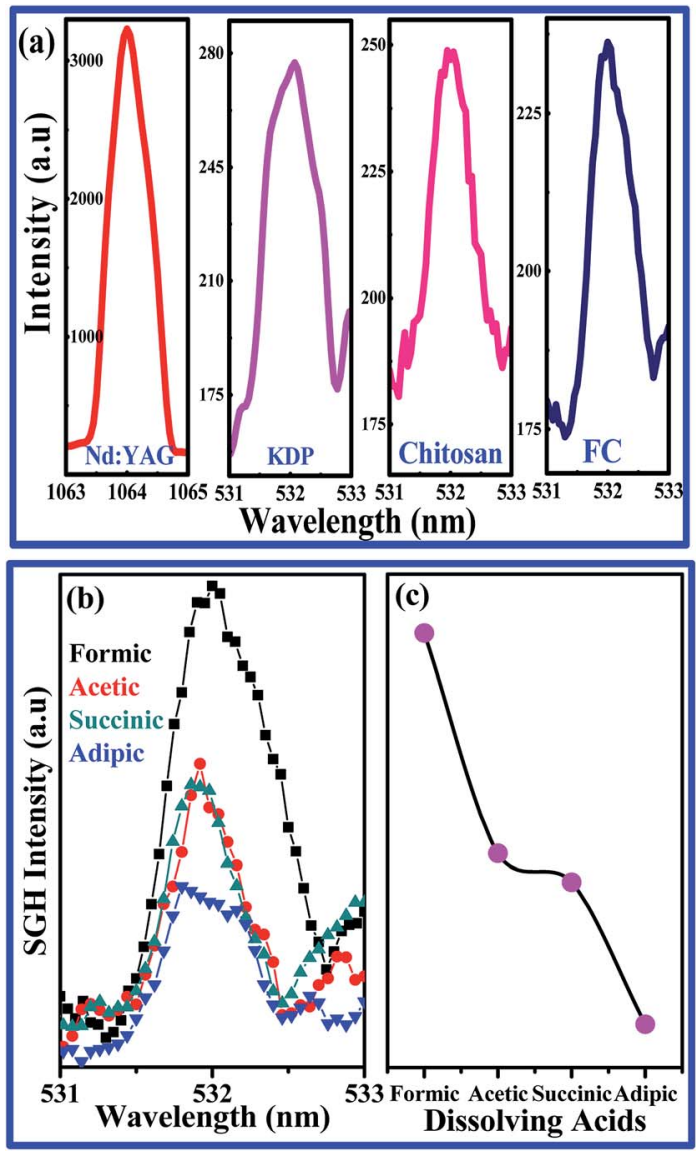

Fig. 3 (a) SHG signals of Nd:YAG source, KDP, chitosan and formate of chitosan (b) normalized SHG intensities and (c) SHG intensity at $532 \mathrm{~nm}$ of chitosan dissolved with formic, acetic, succinic and adipic acids.

pressures as a function of sintering temperature. Since the measured $d_{33}$ values are oscillatory, the most stable values are averaged and are presented in Fig. 4. From the measurement of the $d_{33}$ coefficient, it is observed that up to $330 \mathrm{~K}$, the sample is stable and has a maximum $d_{33}$ coefficient of $18.4 \mathrm{pC} \mathrm{N}^{-1}$ for a load of 5 tons. Further increase in temperature leads to decrease in the $d_{33}$ coefficient, which may due to the disappearance of non-centrosymmetry of chitosan. The measured $d_{33}$ coefficients with the experimental errors are given in Table 2.

\section{Application: chitosan as a vibration sensor}

The existence of non-centrosymmetry and hence the piezoelectricity in the chitosan are confirmed through the abovementioned experiments: SHG and $d_{33}$ measurements. Although it exhibited non-centro symmetry and hence the piezoelectric property, it was tested for suitability in applications such as in vibration sensors. The chitosan samples were also tested for the isolation measurement and mechanical excitation by an $80 \mathrm{~Hz}$ shaker, as given in Fig. 5 a.

Electrical response of chitosan: isolation resistance. If the prepared sensor material is used in a precarious setting, it is very essential to establish an isolation between the sensor and the test material ground. The maximum isolation required for

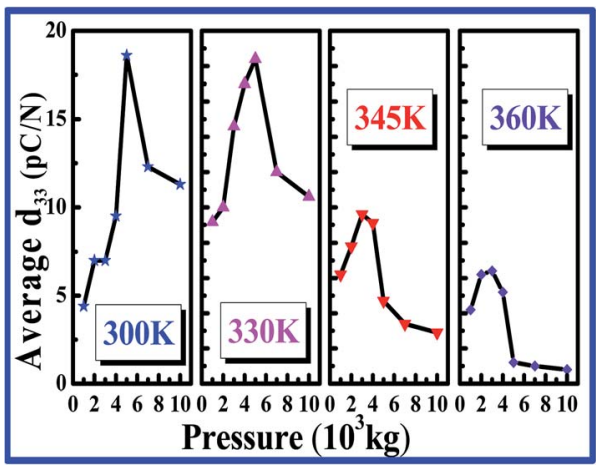

Fig. $4 d_{33}$ coefficient of formate of chitosan for various pressures and sintering temperatures.

safety measurement is only $\sim 100 \mathrm{~V}_{\mathrm{dc}}$. Therefore, if the samples are capable of more than the required isolation, then they can be used as a sensor material for mechanical sensing. The chitosan films (formate, acetate, succinate, and adipate) were electrically characterized using the isolation meter at 50, 100, 250,500 , and $1000 \mathrm{~V}_{\mathrm{dc}}$. The isolation resistance is given in Table 3. It is observed that our samples have very high isolation resistance $(\sim 0.4$ to more than $1 \mathrm{G} \Omega)$ as compared to those obtained in our earlier studies, ${ }^{10,11}$ which confirms the suitability of formate of chitosan for the fabrication of mechanical sensor elements.

Mechanical response of chitosan: detection of $80 \mathrm{~Hz}$ mechanical oscillations. Thin films of chitosan are mounted between the isolation stud and the stainless steel electrode by spot welding technique. The whole setup now acts as a mechanical sensor when it is excited. When mechanical excitation is applied on the electrode disks, it leads to compression and decompression of the chitosan films, which generates charge output based on the magnitude of mechanical excitation. An electrical connection is made to the microdot cable of RG188 standard, and it is attached to the signal conditioner of PCB 481 series for high frequency data acquisition. The top and bottom electrodes are attached with a lead wire for the measurement of charge. This total assembly and the top and bottom disks are isolated from each other by PTFE isolation tape. Hence, the erroneous output from the sensor

Table $2 d_{33}$ coefficient of pellets of chitosan of $1.3 \mathrm{~mm}$ thickness for various temperatures and applied pressures

$\underline{d_{33} \text { coefficient }\left(\mathrm{pC} \mathrm{N}^{-1}\right)}$

\begin{tabular}{|c|c|c|c|c|}
\hline \multirow{2}{*}{$\begin{array}{l}\text { Pressure }\left(10^{3}\right. \\
\left.\mathrm{kg} \text { per } 42.25 \mathrm{~mm}^{2}\right)\end{array}$} & \multicolumn{4}{|c|}{ Temperature (K) } \\
\hline & 300 & 330 & 345 & 360 \\
\hline 1 & $4.4 \pm 0.4$ & $9.2 \pm 0.2$ & $6.2 \pm 0.2$ & $4.2 \pm 0.2$ \\
\hline 2 & $7.0 \pm 0.3$ & $10.0 \pm 0.2$ & $7.8 \pm 0.1$ & $6.2 \pm 0.2$ \\
\hline 3 & $7.0 \pm 0.3$ & $14.6 \pm 0.1$ & $9.6 \pm 0.1$ & $6.4 \pm 0.1$ \\
\hline 4 & $9.5 \pm 0.2$ & $17.0 \pm 0.1$ & $9.1 \pm 0.1$ & $5.2 \pm 0.2$ \\
\hline 5 & $18.6 \pm 0.1$ & $18.4 \pm 0.1$ & $4.7 \pm 0.2$ & $1.2 \pm 0.8$ \\
\hline 7 & $12.3 \pm 0.1$ & $12.0 \pm 0.1$ & $3.4 \pm 0.3$ & $1.0 \pm 0.9$ \\
\hline 10 & $11.3 \pm 0.2$ & $10.6 \pm 0.1$ & $2.9 \pm 0.2$ & $0.8 \pm 1.1$ \\
\hline
\end{tabular}



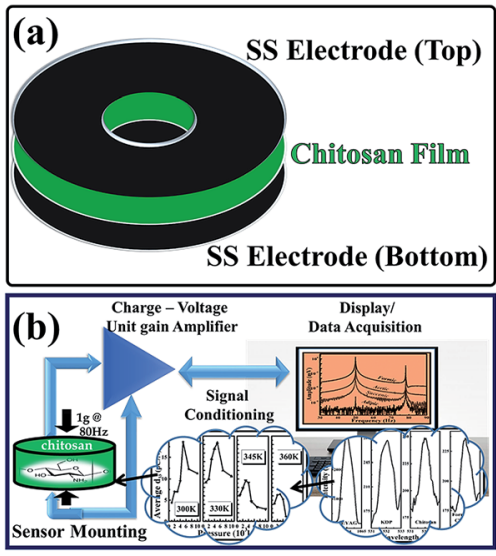

Fig. 5 (a) Chitosan film with stainless steel (SS) electrode plate. (b) Schematic of mechanical excitation measurement and data acquisition.

Table 3 Isolation resistance of thin films of formate, acetate, succinate and adipate of chitosan

\begin{tabular}{|c|c|c|c|c|c|c|}
\hline \multirow[b]{2}{*}{ S. No. } & \multirow{2}{*}{$\begin{array}{l}\text { Sample } \\
\text { legend }\end{array}$} & \multicolumn{5}{|c|}{ Isolation measurement } \\
\hline & & $50 \mathrm{~V}_{\mathrm{dc}}$ & $100 \mathrm{~V}_{\mathrm{dc}}$ & $250 \mathrm{~V}_{\mathrm{dc}}$ & $500 \mathrm{~V}_{\mathrm{dc}}$ & $1000 \mathrm{~V}_{\mathrm{dc}}$ \\
\hline 1 & Formic & $>1 \mathrm{G} \Omega$ & $>1 \mathrm{G} \Omega$ & $401 \mathrm{M} \Omega$ & $516 \mathrm{M} \Omega$ & $500 \mathrm{M} \Omega$ \\
\hline 2 & Acetic & $>1 \mathrm{G} \Omega$ & $>1 \mathrm{G} \Omega$ & $>1 \mathrm{G} \Omega$ & $>1 \mathrm{G} \Omega$ & $>1 \mathrm{G} \Omega$ \\
\hline 3 & Succinic & $>1 \mathrm{G} \Omega$ & $>1 \mathrm{G} \Omega$ & $>1 \mathrm{G} \Omega$ & $>1 \mathrm{G} \Omega$ & $>1 \mathrm{G} \Omega$ \\
\hline 4 & Adipic & $>1 \mathrm{G} \Omega$ & $>1 \mathrm{G} \Omega$ & $>1 \mathrm{G} \Omega$ & $>1 \mathrm{G} \Omega$ & $>1 \mathrm{G} \Omega$ \\
\hline
\end{tabular}

system is eliminated. The schematic of the measurement of mechanical excitation and data acquisition is depicted in Fig. 5b, and with this arrangement, the fabricated sensor films are excited mechanically by the generation of $80 \mathrm{~Hz}$ mechanical oscillations at 1 gravitational acceleration $(g)$ level. Hence, the charge developed in the fabricated chitosan film corresponds to $1 g$, which is almost equivalent to the sensitivity of the sensor element. To confirm the applicability and the realization of chitosan as a sensor, thin films of chitosan are mounted parallel with the commercially available Endevco-make sensor. When the mechanical excitation of $80 \mathrm{~Hz}$ at $1 g$ level is applied, the output of the sensor is fed through a Kiestler-make chargevoltage amplifier with unit gain to the signal conditioner. The acquired output is analyzed by the fast Fourier transform (FFT) algorithm to study the frequency components present in the spectrum. Due to the presence of charge leakage in electronic components and other elements such as power line, the output signal experiences noise at $50 \mathrm{~Hz}$. Hence, the output spectrum consists of both $50 \mathrm{~Hz}$ and $80 \mathrm{~Hz}$ components. FFT spectra of chitosan-based sensors for various acids are shown in Fig. 6 a. While fabricating the sensor, it is very essential to eliminate noise from the output, and the power line leakage at $50 \mathrm{~Hz}$ may be removed by making a filter circuit, as reported by Wang et al. ${ }^{21}$ If the filter circuit is used for the removal of noise from the output spectrum, it may lead to the loss of sensitivity of the sensor and also letdown in detecting mechanical excitation. As an alternative to the abovementioned filter circuit, the sensor could be designed with a hermetical shield, as reported in literature..$^{10,11,22}$

Sensitivity of chitosan dissolved in organic acids. The output signals obtained from the mechanically excited thin films of chitosan dissolved in organic acids and the commercial sensor are out of phase in amplitude. The output of the chitosan based sensor is shown in Fig. $6 \mathrm{~b}$ and the phase shifts are compared with those of the commercial sensor output, which is depicted in Fig. 6c. While studying the effect of dissolution of chitosan

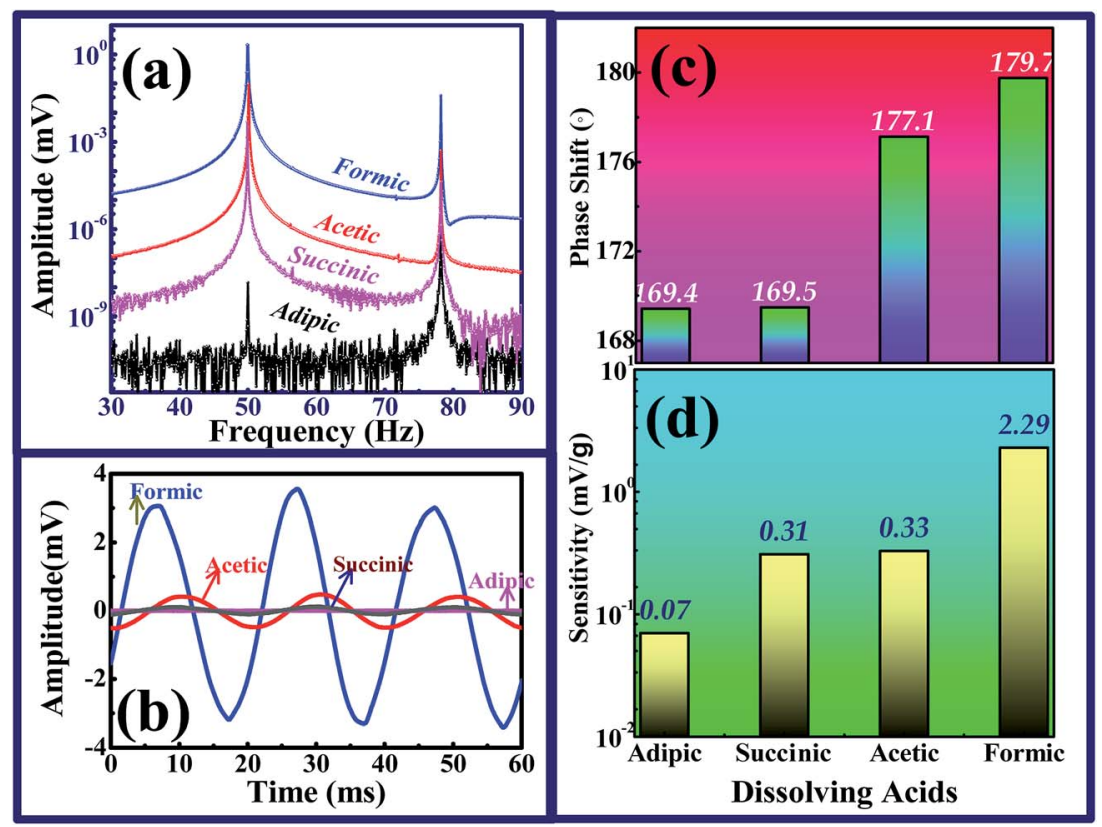

Fig. 6 (a) FFT spectra, (b) output amplitude, (c) phase shift and (d) sensitivity of chitosan based sensor. 
on these acids, the sensitivity of formate of chitosan is quite high. Hence, formic acid is endorsed to be the most appropriate acid for sensor fabrication. The sensitivity of various dissolving acids of chitosan is depicted in Fig. $6 \mathrm{~d}$.

\section{Conclusion}

In the present study, an attempt was made to investigate the existence of non-centrosymmetry of a biopolymer, namely chitosan, through XRD analysis and second harmonic generation experiments. From the SHG experiment, the frequency splitting was observed at $532 \mathrm{~nm}$ with the $1064 \mathrm{~nm} \mathrm{Nd:YAG} \mathrm{source,}$ which confirmed the non-centrosymmetry nature of the chitosan samples due to which the chitosan exhibited piezoelectric property. Temperature-dependent $d_{33}$ coefficients for various pressures confirmed the existence of piezoelectricity. Based on the abovementioned results, chitosan was dissolved in various organic acids such as formic, acetic, succinic, and adipic, and the suitability of chitosan film for vibration sensors was studied. Compared with other acids, films prepared by dissolving chitosan in formic acid provided good sensitivity for the sensor. From the isolation resistance measurement, the fabricated sensor films were established to have a very high isolation $(\sim 0.4$ to more than $1 \mathrm{G} \Omega$ ) suitable for precarious condition, whereas the explosive environment need isolation only up to $100 \mathrm{M} \Omega$. However, the excitation vibration is $80, \mathrm{~Hz}$ at $1 g$, and the output signals are developed with a noise at $50 \mathrm{~Hz}$ due to power line leakage. However, the fabrication of the sensor causes a leakage that may be removed by hermitic shielding. All the abovementioned investigations reveal the suitability of chitosan films in vibration sensors.

\section{Acknowledgements}

Authors K. Jayakumar and E. Praveen thank Indian Space Research Organization - India for the financial support under the RESPOND program.

\section{References}

1 M. Rinaudo, Prog. Polym. Sci., 2006, 31, 603-632.

2 M. Ioelovich, Res. Rev.: J. Chem., 2014, 3, 7-14.

3 I. G. Lalov, I. I. Guerginov, M. A. Krysteva and K. Fartsov, Water Res., 2000, 34, 1503-1506.
4 M. Vakili, M. Rafatullah, B. Salamatinia, A. Z. Abdullah, M. H. Ibrahim, K. B. Tan, Z. Gholami and P. Amouzgar, Carbohydr. Polym., 2014, 113, 115-130.

5 Y. Zhang and M. Zhang, J. Biomed. Mater. Res., 2001, 55, 304312.

6 D. Archana, J. Dutta and P. K. Dutta, Int. J. Biol. Macromol., 2013, 57, 193-203.

7 R. Jayakumar, M. Prabaharan, P. T. Sudheesh Kumar, S. V. Nair and H. Tamura, Biotechnol. Adv., 2011, 29, 322-337.

8 S. Torrado, P. Prada, P. M. De La Torre and S. Torrado, Biomaterials, 2004, 25, 917-923.

9 A. S. Sombra, C. C. Silva, C. G. A. Lima, A. G. Pinheiro, J. C. Goes and S. D. Figueiro, Phys. Chem. Chem. Phys, 2001, 3, 4154-4157.

10 S. Murugan, D. Karthikesan, S. Sellathurai, E. Praveen and K. Jayakumar, Fifth International Symposium on Electronic System Design (ISED) Surathkal, 2014, pp. 199-202.

11 E. Praveen, S. Murugan and K. Jayakumar, AIP Conf. Proc., 2015, 1665(050032), 1-3.

12 K. Ogawa and S. Inukai, Carbohydr. Res., 1987, 160, 425-433.

13 A. Pawlak and M. Mucha, Thermochim. Acta, 2003, 396, 153166.

14 K. Ogawa, S. Hirano, T. Miyanishi, T. Yui and T. Watanabe, Macromolecules, 1984, 17, 973-975.

15 N. Cartier, A. Domard and H. Chanzy, Int. J. Biol. Macromol., 1990, 12, 289-294.

16 E. Arnold, D. M. Himmel and M. G. Rossmann, Basic Crystallography, in International Tables for Crystallography Volume F: Crystallography of biological macromolecules, ed. J. Drenth, Kluwer Academic Publishers, Netherland, 2006, ch. 2.1, pp. 45-63.

17 T. H. Hahn, Contents and arrangement of the tables, in International Tables for Crystallography, Volume A: Space Group Symmetry, ed. T.H. Hahn and A. Looijenga-Vos, 2006, ch. 2.2, pp. 17-41.

18 Z. Osman and A. Arof, Electrochim. Acta, 2003, 48, 993-999.

19 A. Zajac, J. Hanuza, M. Wandas and L. Dymińska, Spectrochim. Acta, Part A, 2015, 134, 114-120.

20 A. Sionkowska, Biomaterials, 2004, 25, 795-801.

21 Y. S. Wang, Y. X. Gai and S. M. Li, 2011 Int. Conf. Control. Autom. Syst. Eng. CASE 2011, 2011, pp. 2-5.

22 S. Murugan, M. V. N. Prasad and K. Jayakumar, AIP Conf. Proc., 2016, 1731(050003), 1-3. 